\title{
ON TOPOLOGIES OVER RINGS
}

\author{
SYED M. FAKHRUDDIN \\ Department of Mathematics \\ University of Petroleum and Minerals \\ Dhahran, Saudi Arabia \\ (Received February 4, 1983 and in revised form June 24, 1983)
}

ABSTRACT. In this note, we show that if a topology $\underline{F}$ over a ring $A$ satisfies a certain finiteness condition, then the Gabriel topology $\underline{G}$ generated by $\underline{F}$ can be explicitly constructed and it also satisfies the same finiteness condition.

KEY WORDS AND PHRASES. Filter, Gabriel topology, torsion theory, torsion ideal. 1980 MATHEMATICS SUBJECT CLASSIFICATION CODE. 16 A08

1. INTRODUCTION.

Let $A$ be an associative ring with identity. A filter of right ideals $F$ of $A$ is called a right topology (a pretopology in Stenstrom (1971)), if it is closed for right quotients by arbitrary elements of A. Suppose $F$ satisfies in addition: If $I$ is a right ideal of $A$ such that ( $I: a) \in \underline{F}$ for every a $\varepsilon J$ and $J \in \underline{F}$ then I $\varepsilon \underline{F}$ : then $\underline{F}$ is called a right Gabriel topology over $A$ (an additive topology in Stenstrom (1971)).

In [1], it is remarked that "if $\underline{E}$ is a pretopology, then $J(E)$ - the weakest topology containing $\underline{E}$ - is the topology corresponding to the heriditary torsion theory generated by $\{\mathrm{A} / \mathrm{I} \mid \mathrm{I} \in \underline{\mathrm{F}}\}$ ".

In this note, we constructed explicitly $J(\underline{E})$ from $\underline{E}$, without recourse to the torsion theory, provided $\underline{E}$ satisfies a rather natural finiteness condition. It turns out that $J(\underline{E})$ also satisfies this condition. Moreover, this condition holds for all topologies over a right Noetherian ring.

Hereafter, by a ring we mean an associative ring with identity, and ideal means right ideal; hence, all topologies are right topologies.

2. CONSTRUCTION.

Let $F$ be a topology over a ring $A$ and $I$ an ideal. We define the quotient of I with respect to $\underline{F}$ to be

$$
(\underline{F}: I)=\{a \mid a \varepsilon A:(I: a) \varepsilon \underline{F}\} \text {. }
$$

One checks easily that (F : I) is an over-ideal of $I$ and it is equal to A precisely when $I \varepsilon F$ and that this quotient operation is monotonic in each of the variables. 
Let us write $I_{1}$, instead of $(\underline{F}: I)$ and define inductively $I_{n+1}=\left(I_{n}\right)$, and finally $\overline{\mathrm{I}}=\bigcup_{n \in N} I_{n}$. Then $\overline{\mathrm{I}}$ is an (union of an ascending chain of) idea1 containing $I$, called the closure of $I$ (with respect to $\underline{E}$ ).

It is straightforward to verify that if $I \in \underline{F}$ then $\bar{I}=I_{1}=A$ and it is a preclosure operation in the topological sense, namely $I \subseteq J$ implies $\overline{\mathrm{I}} \subseteq \overline{\mathrm{J}}$ and $\overline{\mathrm{I} \cap \mathrm{J}}=\overline{\mathrm{I}} \cap \overline{\mathrm{J}}$ for any two ideals $\mathrm{I}$ and $\mathrm{J}$ of $\mathrm{A}$.

Moreover, since $(\underline{F}:(I: A))=((\underline{F}: I): a)$ as one can check easily, we conclude also that $(\overline{(I: a)}=(\bar{I}: a)$ for an element a $\varepsilon A$.

A topology $\underline{F}$ over $A$ is called sequence-finite, if $\left\{\mathrm{I}_{n}\right\}_{n \in N}$ is an ascending sequence of ideals of $A$ such that their union belongs to $\underline{F}$, then $I_{n}$ is a member of $\underline{F}$ for some $n$.

Suppose the ring $A$ has the property that every topology has, a subbase of finintely generated ideals -- in particular, if A is Noetherian -- then all topologies over $A$ are sequence-finite [1].

In the case of a sequence-finite topology $\underline{F}$, the remarks in the preceding paragraph concerning the closure of an ideal can be sharpened. Indeed one has that for an ideal $I$ of $A, \bar{I} \varepsilon \underline{F}$ if $I_{n} \varepsilon \underline{F}$ for some $n$ and the closure of the union of an ascending sequence of ideals equals the union of the closures of the members of the chain and consequently, the closure operation is in effect idempotent; namely $\overline{\bar{I}}=\bar{I}$ for an ideal $I$ of $A$.

Now we have

THEOREM 1. Let $A$ be a ring with identity and $\underline{F}$ a sequence-finite topology over A - Then the Gabriel topology $G$ generated by $\underline{F}$ is given by

$$
\underline{G}=\{I \mid I \text { is an ideal of } A: \bar{I} \varepsilon \underline{F}\}
$$

and $\underline{G}$ is also sequence finite.

PROOF. That $\underline{G}$ is a topology follows easily from the preceding discussion.

Suppose now that $I$ is an ideal of $A$ such that for some $J$ in $\underline{F}$ and for every $a \in J$, we have (I:A) $\varepsilon \underline{F}$. Then $(I: a)=(\bar{I}: a) \varepsilon \underline{F}$ for every a in $\mathrm{J}$. Consequently, $G \subseteq(\underline{F}: \overline{\mathrm{I}}) \subseteq \overline{\overline{\mathrm{I}}}=\overline{\mathrm{I}}$. Hence $\overline{\mathrm{I}} \varepsilon \underline{G}$. But then $\overline{\overline{\mathrm{I}}}=\overline{\mathrm{I}} \varepsilon \underline{F}$, so that $I \in \underline{G}$ also, showing $\underline{G}$ is a Gabriel topology.

Suppose $\left\{I_{n}\right\}_{n \in N}$ be an ascending sequence of ideals such that $U I_{n}$ belongs to $\underline{G}$ - Then $\overline{U I_{n}}$ belongs to $\underline{F}$. Now the sequence finiteness of $\underline{F}$ and the definition of $\underline{G}$ implies that $I_{n}$ belongs to $\underline{G}$ for some $n$.

Let $\underline{H}$ be a Gabriel topology containing $\underline{F}$ and $I$ an ideal belong to $\underline{G}$, then $\overline{\mathrm{I}}$ and so $I_{n}$ belongs to $\overline{\mathrm{F}}$ for some $n$. Therefore $I_{n} \varepsilon \underline{H}$. Since $\underline{H}$ is a Gabriel topology, $I_{n-1}$ and consequently $I_{0}=1$ belongs to $\underline{H}$. Thus $\underline{G} \subseteq \underline{H}$.

The construction above shows that two sequence-finite topologies $\underline{F}$ and $\underline{G}$ generate the same Gabriel topology iff for every $I$ in $\underline{F}, \overline{\mathrm{I}}^{G}$ (the closure of I with respect to $G$ ) belongs to $\underline{G}$ and vice-versa.

Also, if $A$ is a Noetherian ring then all Gabriel topologies can be constructed using the above method. 
In conclusion, we note that without the sequence-finite assumption, the Gabriel topology $\underline{G}$ generated by $\underline{F}$ can be described by a transfinite process as follows: Let $\underline{F}_{1}=\left\{I \mid I\right.$ an ideal and $\left.I_{1}=(\underline{F}: I) \varepsilon \underline{F}\right\}$. The $\underline{F}_{1}$ is a topology containing $\underline{F}$. For a transfinite ordinal $\propto$ we define

$$
\underline{F}_{\alpha}=\quad\left\{\begin{array}{ll}
\left(\mathrm{F}_{\mathrm{B}}\right)_{1} & \text { if } \alpha=\beta+1 \\
{ }_{3<\alpha}^{\prime} \mathrm{F}_{\beta} & \text { otherwise }
\end{array} .\right.
$$

Then there exists an ordinal $\delta$ such that $\left(\underline{F}_{\delta}\right)_{1}=\underline{F}_{\delta}$ and $\underline{F}_{\delta}$ is then the desired Gabriel topology generated by $\underline{F}$.

Our construction above shows that in the presence of sequence-finiteness $\delta$ is equal to " .

ACKNOWLEDGEMENT. This work was done during a hospitable stay at Tulane University, New Orleans (LA.), while on sabbatical leave with full financial support from the University of Petroleum and Minerals, Dhahran (Saudi Arabia).

\section{REFERENCES}

1. STENSTROM, B. Rings and Modules of Quotients, Springer Lecture notes in Mathematics V. 237, 1971 .

2. STENSTROM, B. Rings of Quotients, Springer-Verlag-Heidelberg, Ground Lehren-Band V. 217,1975 . 


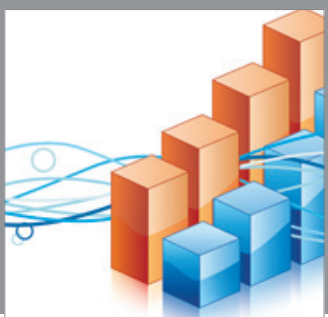

Advances in

Operations Research

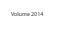

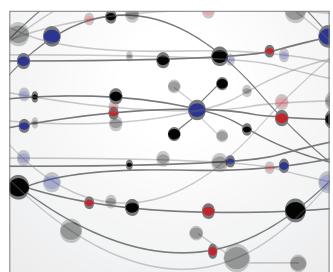

\section{The Scientific} World Journal
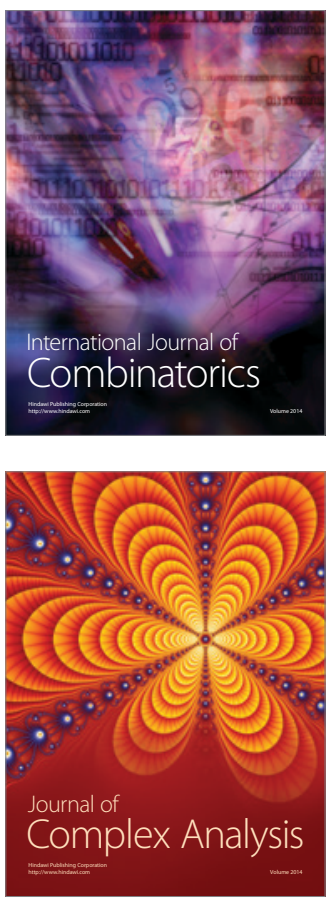

International Journal of

Mathematics and

Mathematical

Sciences
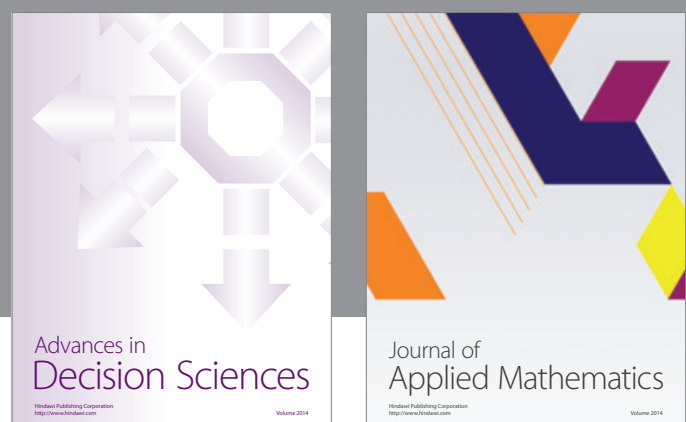

Journal of

Applied Mathematics
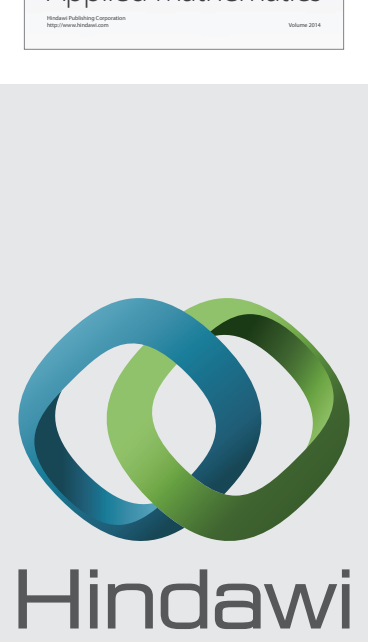

Submit your manuscripts at http://www.hindawi.com
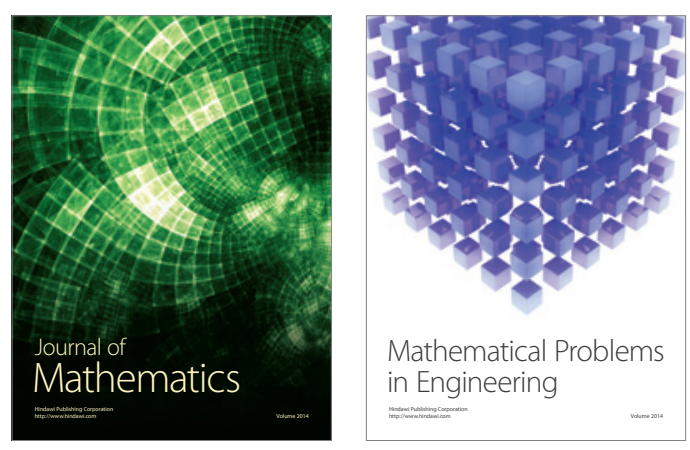

Mathematical Problems in Engineering
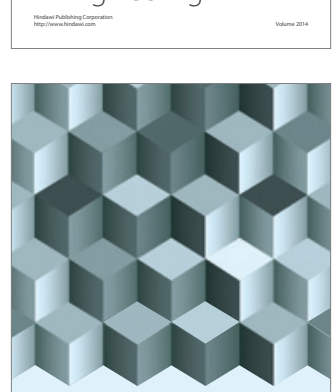

Journal of

Function Spaces
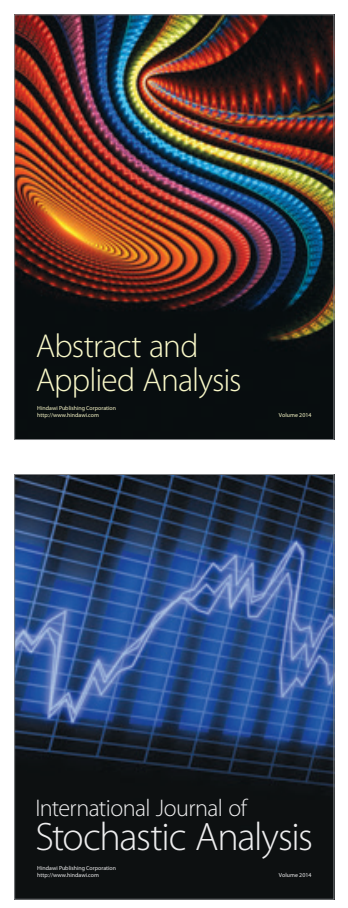

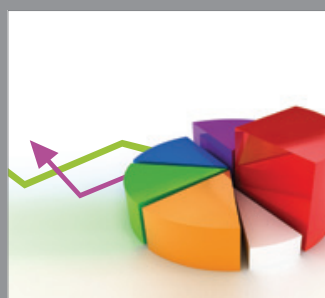

ournal of

Probability and Statistics

Promensencen
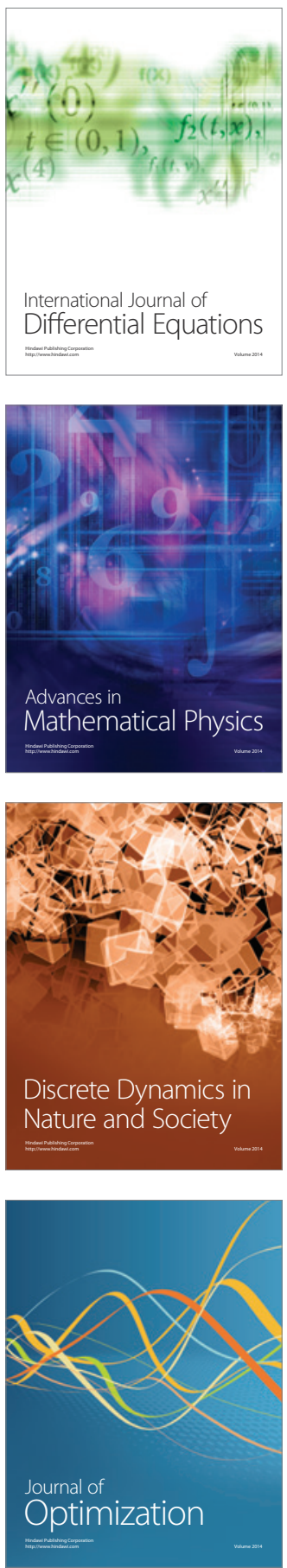